\title{
Los elementos facilitadores de la comprensión oral empleados por el profesor del aula regular con adolescentes sordos.
}

\section{Jesús Valero}

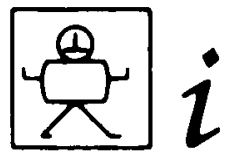

¿Qué ocurre cuando se integra un alumno-a sordo-a en un aula normalizada? ¿Qué bace el profesor y cómo influyen sus competencias en la educación? ¿Qué cambios realiza para afrontar la nueva situación?

Esta investigación analiza esos cambios (en general positivos tanto para el-la niño-a sordo-a como para los compañeros) y las competencias que convendría facilitar el docente.

\section{PLANIFICACION DEL ESTUDIO}

El tratamiento educativo del alumno sordo ha presentado importantes modificaciones en los últimos años. Actualmente, la mayor parte de los estudiantes sordos permanecen escolarizados en centros públicos regulares, recibiendo el apoyo de un logopeda o profesor especializado en el propio centro.

En este sentido, la integración se concibe como un proceso encaminado a la utilización de procedimientos educativos que incidan tanto sobre la adaptación de contenidos como sobre la utilización de elementos que mejoren el desarrollo del sordo en las áreas comunicativa y social.

En nuestro país, la mayoría de los trabajos realizados para analizar las actitudes del profesor hacia la educación diferenciada coinciden en destacar la importancia que la conceptualización de su propia labor como docente, las expectativas sobre el alumno y la experiencia previa en integración mantienen en relación a la implicación del docente en el momento de adoptar determinadas modificaciones y estrategias educativas diferenciadas (García y Alonso, 1985; Monereo y Guardia, 1987; Parrilla, 1988; Aguilera, 1990; Dengra et al., 1991).

Por su parte, el adolescente sordo precisa de estas estrategias en un intento por superar satisfactoriamente las diferentes situaciones comunicativas generadas en los contextos académicos. Esta necesidad suele obligar al profesor a rea- 
lizar diversas adaptaciones tanto pedagógicas como comunicativas, respecto a las actividades más corrientes realizadas en el aula (Silvestre, 1991).

No obstante, también es frecuente que el estudiante sordo no explicite sus dificultades de comprensión. En este sentido, la experiencia nos indica como frente a un comentario del oyente el sujeto sordo suele aparentar comprenderlo, cuando en realidad su comprensión ha sido casi nula.

A lo largo de la pasada década fueron relativamente frecuentes los estudios centrados en las adaptaciones lingüísticas del profesor respecto al alumno sordo en el marco tanto del aula especial como del aula regular (Huntington y Watton, 1981, 1982; Hegarty et al., 1986; Weiss, 1986; Saur et al. 1987; Hartley y Arnold, 1988). La revisión de sus aportaciones más significativas pone de manifiesto las dificultades que para el sordo presenta la comunicación en el aula, mostrándose por regla general el adulto más controlador y directivo en las interacciones con el sordo del que suele ser habitual respecto al oyente.

La mayoría de estos trabajos, no obstante, inciden básicamente en el análisis del formato de la expresión oral, olvidando que la naturaleza específica de los actos comunicativos atípicos entre varios participantes sometidos a reglas y convenciones diferenciadas sugieren la necesidad de un enfoque pragmático, donde se contemplen asimismo las intencionalidades de los hablantes/oyentes.

La sociolingüística ha destacado cómo a cada contribución discursiva el participante más experto debe facilitar las pistas necesarias para eliminar en lo posible los errores de interpretación del participante menos experimentado. En base a estos criterios, y desde una perspectiva pragmática del lenguaje aplicada a la comunicación con el deficiente auditivo, cabe destacar las últimas investigaciones del Wood et al. (1986); Lynas (1986); Kretschmer y Kretschmer (1989); Power, Wood y Wood (1990).

Por nuestra parte desde hace dos años pretendemos analizar y establecer una posible relación entre las estrategias de adaptación empleadas por el profesor y un conjunto de variables intrapersonales y contextuales presentes durante las interacciones en el aula regular. No obstante, somos conscientes de que los procesos interactivos no constituyen ningún esquema lineal, en el sentido que a un comportamiento ha de continuar de forma automática un determinado resultado en el alumno. La influencia educativa de dichos procesos se ejerce afortunadamente a través de situaciones mucho más complejas, donde la actividad constructiva del alumno se manifiesta abiertamente como uno de los factores determinantes del éxito educativo.

En este artículo se exponen los primeros datos obtenidos mediante una serie de cuestionarios y entrevistas personales realizadas a un grupo de adolescentes sordos profundos y a aquellos profesores considerados por los propios alumnos como más/menos fácilmente inteligibles durante el desarrollo de sus clases. Estos datos suponen la primera aproximación de un estudio más amplio donde fue filmada la actividad académica ordinaria de diez aulas integradoras con el fin de analizar las estrategias específicas empleadas por el profesor en sus interacciones con el estudiante sordo.

\section{POBLACION Y PROCEDIMIENTO}

Por un lado, la muestra de sujetos estudiados la conforman 24 adolescentes sordos integrados en diferentes centros escolares regulares de Cataluña. Todos ellos presentaban el siguiente perfil: 
1. Estar afectado de sordera profunda prelocutiva (pérdida media de $90 \mathrm{~dB}$. o superior en ambos oídos).

2. No presentar ningún trastorno asociado conocido.

3. Tener una edad comprendida entre los 12 y los 17 años.

4. Estar escolarizado en un centro preferiblemente público, en los niveles de Segunda etapa de EGB o de Secundaria.

5. Mantener una escolaridad en régimen de integración en aulas de oyentes.

Para determinar el grado de comprensión verbal del alumno sordo se procedió al empleo adaptado de una prueba inspirada en Jensema et al. (1978), citada por Marchesi (1987). Se solicitó a los logopedas de cada alumno que establecieran su nivel de comprensión verbal en base a una de estas categorías:

I. Comprende únicamente aquellos elementos principales del enunciado.

II. Comprende sólo enunciados simples, sin establecer relaciones entre ellos.

III. Comprende enunciados más complejos relacionados por elementos de causalidad.

IV. Prácticamente comprende cualquier tipo de enunciado, a excepción de los que establecen relaciones complejas de subordinación o voz pasiva.

V. No presenta especiales problemas de comprensión lingüística, mientras se le anticipe el léxico desconocido.

Las características de la muestra de estudiantes sordos quedan reflejadas en la Tabla I.

TABLA I

Principales características de los alumnos sordos pertenecientes a la muestra $(N=24)$.

\begin{tabular}{|c|c|c|c|c|}
\hline ALUMNO & EDAD & SEXO & CURSO & $\begin{array}{c}\text { NIVEL } \\
\text { COMPRENSION }\end{array}$ \\
\hline 1 & 12 & $\mathrm{H}$ & $6^{\circ} \mathrm{EGB}$ & IV \\
\hline 2 & 14 & $\mathrm{H}$ & 6. $\mathrm{EGB}$ & III \\
\hline 3 & 13 & $\mathrm{H}$ & $60^{\circ} \mathrm{EGB}$ & III \\
\hline 4 & 14 & $\mathrm{H}$ & $6 .^{\circ} \mathrm{EGB}$ & III \\
\hline 5 & 14 & $\mathrm{H}$ & $6^{\circ} \mathrm{EGB}$ & IV \\
\hline 6 & 13 & $\mathrm{~V}$ & $60^{\circ} \mathrm{EGB}$ & III \\
\hline 7 & 12 & V & 6. $\mathrm{EGB}$ & III \\
\hline 8 & 14 & $\mathrm{H}$ & $60^{\circ} \mathrm{EGB}$ & II \\
\hline 9 & 14 & $\mathrm{H}$ & 7. $\mathrm{EGB}$ & I \\
\hline 10 & 14 & $\mathrm{H}$ & 7. $\mathrm{EGB}$ & II \\
\hline 11 & 14 & $\mathrm{H}$ & 7. $\mathrm{EGB}$ & II \\
\hline 12 & 14 & $\mathrm{~V}$ & $8 .^{\circ} \mathrm{EGB}$ & III \\
\hline 13 & 16 & $\mathrm{H}$ & 8. $\mathrm{E} G \mathrm{~B}$ & IV \\
\hline 14 & 16 & $\mathrm{H}$ & $8 .^{\circ} \mathrm{EGB}$ & III \\
\hline 15 & 14 & $\mathrm{~V}$ & 8. ${ }^{\circ} \mathrm{EGB}$ & IV \\
\hline 16 & 14 & V & $8 .^{\circ} \mathrm{EGB}$ & III \\
\hline 17 & 14 & $\mathrm{H}$ & 8. ${ }^{\circ} \mathrm{EGB}$ & IV \\
\hline 18 & 15 & V & $10^{\circ} \mathrm{BUP}$ & III \\
\hline 19 & 15 & V & $1 .^{\circ} \mathrm{BUP}$ & IV \\
\hline 20 & 15 & V & $1 .^{\circ} \mathrm{BUP}$ & V \\
\hline 21 & 17 & $\mathrm{H}$ & $10^{\circ} \mathrm{FP}$ & III \\
\hline 22 & 16 & $\mathrm{H}$ & $2 .^{\circ} \mathrm{BUP}$ & IV \\
\hline 23 & 16 & $\mathrm{H}$ & 2. BUP & IV \\
\hline 24 & 17 & $\mathrm{H}$ & $2 .^{\circ} \mathrm{FP}$ & III \\
\hline
\end{tabular}

En el momento de realizar el estudio, un total de 20 alumnos $(83,3 \%)$ permanecían entre 6 y 10 años integrados en sus respectivos centros; siendo el 
promedio de permanencia en el centro regular del resto de los alumnos estudiados todavía más elevado, a excepción de los alumnos que cursan sus estudios en Secundaria.

Por su parte, el resto de la muestra analizada lo configuraban un total de 43 enseñantes; 24 de ellos fueron considerados por los alumnos como profesores inteligibles durante sus exposiciones en clase y 19 como poco inteligibles. La mayoría de todos ellos $(60,5 \%)$ no habían tenido ningún tipo de experiencia previa directa con el alumno sordo; un $23,25 \%$ poseían la experiencia de un único curso escolar; un $11,6 \%$ de dos cursos y únicamente dos profesores $(4,65 \%)$ habían trabajado con el alumno sordo tres cursos, si bien éstos normalmente no habían sido consecutivos.

Las dimensiones analizadas en esta primera fase del estudio fueron:

1. Las principales dificultades que comporta el trabajo didáctico cotidiano con el alumno sordo a juicio de cada profesor.

2. Las modificaciones estructurales efectuadas en el aula como consecuencia de la presencia de un alumno sordo en ella.

3. Las ayudas que recibe el alumno como complemento al trabajo en el área de aprendizaje estudiada.

4. Los principales receptores de las demandas de ayuda del alumno sordo, en situaciones de incomprensión de contenidos en el aula.

5. Los facilitadores empleados por el profesor para solucionar las situaciones de incompresión generadas en el aula respecto al alumno sordo.

En primer lugar, a excepción de la primera dimensión todas las restantes fueron analizadas diferenciando los datos recogidos a partir de las respuestas de los profesores considerados como más fácilmente comprensibles en sus exposiciones en clase de aquellos que no lo son.

Además, las dimensiones tercera, cuarta y quinta fueron analizadas desde la perspectiva de lo que opinaba el profesor y de lo que opinaba el propio alumno, con la finalidad de conocer hasta qué punto existía coincidencia de criterios entre ambos interlocutores o, por el contrario, existía disparidad en sus valoraciones.

\section{RESULTADOS}

El análisis efectuado de los datos obtenidos es de tipo descriptivo, en un intento por examinarlos y poseer una orientación inicial sobre cada una de las dimensiones estudiadas.

\section{Principales dificultacies en el trabajo didáctico con el estudiante sordo}

Se han analizado estas dificultades agrupándolas en tres áreas:

A. Dificultades referidas a los contenidos académicos.

B. Dificultades referidas a los procedimientos didácticos empleados.

C. Dificultades referidas al déficit o a la actitud del propio alumno.

Tal como aparece reflejado en la Figura 1, mayoritariamente los profesores entrevistados centran el foco de sus problemas respecto al alumno sordo en los procedimientos de trabajo en el aula: «la compaginación de varias actividades pa- 
ralelas —sordo/oyentes—»; «la necesidad de una mayor dedicación individualizada»; «la no transgresión de las reglas comunicativas básicas respecto al alumno sordo»

En segundo término, destacan las dificultades centradas en los contenidos académicos: «... tiene dificultades para aprender las nociones más abstractas de la materia»; «(el alumno) no posee suficiente vocabulario, lo que le impide seguir el ritmo de la clasess...

Y por último, destacan las dificultades referidas al propio déficit o a la actitud del alumno: «... no suele tener interés por el trabajo»; «no tiene bábitos de estudio autónomos»; "no sé si utiliza la sordera como excusa»...

FIGURA 1

\begin{tabular}{|l|r|c|c|c|}
\hline \multirow{2}{*}{ DIFICULTADES } & \multicolumn{4}{|c|}{ EXPERIENCIA CON EL ALUMNO } \\
\cline { 2 - 5 } & 0 AÑOS & 1 AÑO & 2 AÑOS & 3 ANOS \\
\hline Contenido & 10 Prof. & 3 Prof. & 1 Prof. & - \\
Procedimiento & 8 Prof. & 4 Prof. & 3 Prof. & 2 Prof. \\
Sordera & 8 Prof. & 2 Prof. & 1 Prof. & - \\
\hline TOTAL & 26 Prof. & 9 Prof. & 5 Prof. & 2 Prof. \\
\hline
\end{tabular}

Principales dificultades explicitadas por el profesor $(N=42)$.

Resulta interesante observar la modificación de estos valores en función de la experiencia previa del profesor respecto al alumno. De este modo, se observa la existencia de una relación inversamente proporcional entre la experiencia y las dificultades centradas tanto en los contenidos como en el propio déficit.

En tal sentido podemos conjeturar cómo a medida que aumenta la experiencia del profesor aumenta el interés por conocer estilos didácticos de trabajo que resulten más funcionales para el alumno, siendo cada vez menores los temores y las dudas frente a sus posibilidades en el aula regular como consecuencia del inconveniente auditivo. Conviene, no obstante, considerar la autovaloración que el profesor mantiene sobre su tarea respecto al alumno sordo, como una variable que puede acelerar o inhibir este proceso.

\section{Modificaciones estructurales ejercidas en el aula}

Mayoritariamente, los profesores no suelen efectuar modificación alguna en el aula únicamente por el hecho de tener un alumno sordo integrado en la clase $(54,5 \%)$; sólo efectúan algún tipo de modificaciones un $38,5 \%$ de profesores, y dichas modificaciones son sustanciales tan sólo en un $7 \%$ del total de la muestra.

Por orden decreciente, estas modificaciones estructurales afectan:

1. El sistema de evaluación.

2. La elección de contenidos.

3. La secuenciación de las unidades didácticas.

4. La determinación de los conceptos básicos del área de conocimientos trabajada.

5. La elección de materiales didácticos.

6. La adecuación del espacio físico en el aula.

No se ha encontrado ninguna diferencia significativa entre las respuestas 
vertidas por los profesores considerados por los alumnos como más inteligibles, de las vertidas por los considerados como menos inteligibles.

Por todo ello, estos resultados podrían indicar la necesidad de incidir en el grado de información que el profesorado mantiene sobre la adopción de ajustes pedagógicos específicos con alumnos sordos. No obstante, debemos ser prudentes a la hora de valorar los resultados en esta dimensión, puesto que a nuestro juicio podrían existir tres circunstancias que inciden sobre la adopción de ajustes estructurales en el aula: A) El tipo de Programación Curricular seguido por el alumno. B) El estilo pedagógico del profesor (progresista/tradicional). C) La información que sobre la especificidad educativa del sordo posee el profesor. De este modo, si la forma habitual de proceder del profesor encaja con los principios didácticos más apropiados al alumno sordo -educación vivencial e individualizada - no será necesario cambiar sustancialmente la dinámica cotidiana del aula.

\section{Las ayudas complementarias que recibe el alumno}

A la hora de analizar los datos obtenidos en esta dimensión es preciso diferenciar entre aquellas ayudas que persiguen la anticipación de contenidos con anterioridad al desarrollo del trabajo en clase de aquellas otras posteriores a clase.

El 62,5\% de los alumnos afirman no poseer ningún tipo de refuerzo previo, frente a un $37,5 \%$ que afirma recibir información con anterioridad al trabajo del aula, si bien esta tarea de anticipación no suele ser constante. Mayoritariamente la anticipación de contenidos la suelen realizar o bien el logopeda o bien el profesor de apoyo.

Sin embargo, prácticamente la totalidad de los alumnos sordos reciben un refuerzo en sus aprendizajes posterior a la actividad del aula, siendo las personas que principalmente propinan este refuerzo el logopeda, el profesor del aula regular y la familia (ver Figura 2).

Figura 2

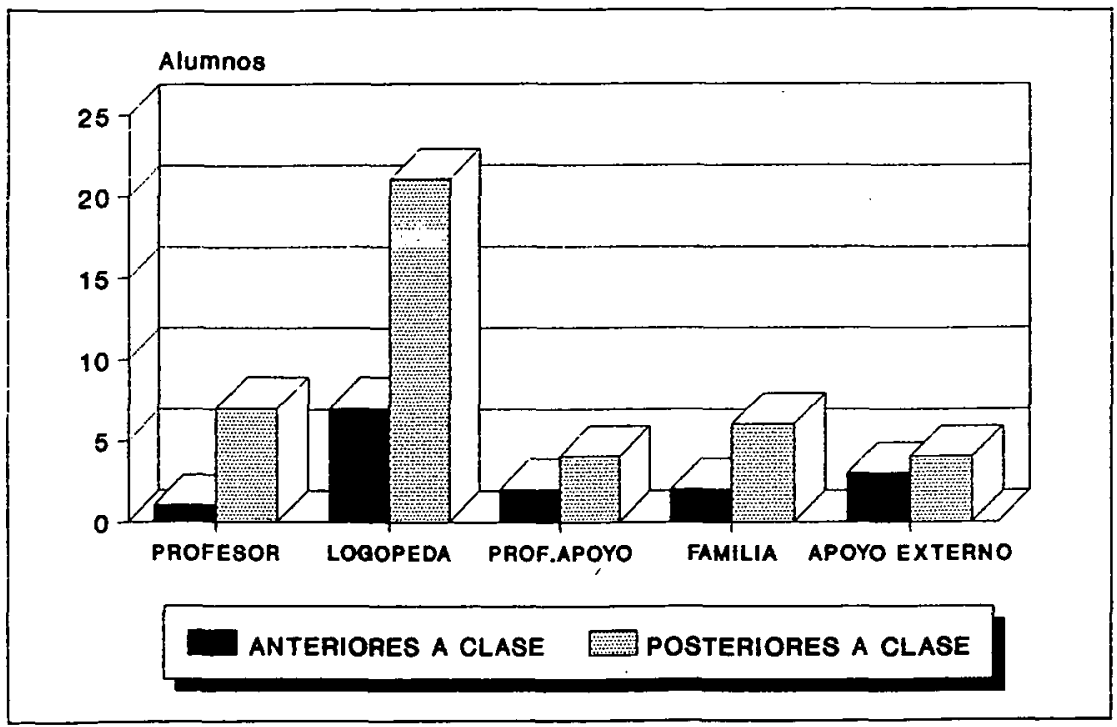

Distribución de las ayudas complementarias que recibe el alumno sordo 
Junto a esta dimensión, parece necesario conocer tanto el tipo de ayuda que recibe el alumno como el área de conocimientos en la que mayormente recibe este tipo de ayudas. En este sentido hemos encontrado una singular distribución a la hora de valorar los propios alumnos sordos al profesor que les resulta más comprensible en sus explicaciones orales (ver Figura 3).

Figura 3

\begin{tabular}{|c|c|c|c|c|c|c|c|}
\hline \multirow{2}{*}{$\begin{array}{c}\text { Grado } \\
\text { comprensión }\end{array}$} & \multirow[t]{2}{*}{ Materias } & \multicolumn{5}{|c|}{$\begin{array}{l}\text { Nivel comprensión } \\
\text { alumno }\end{array}$} & \multirow[t]{2}{*}{ Total } \\
\hline & & $I$ & II & III & IV & $\mathrm{V}$ & \\
\hline ALTO & $\begin{array}{l}\text { Matemát. } \\
\text { Sociales } \\
\text { Naturales } \\
\text { Lengua }\end{array}$ & $\frac{-}{1}$ & $\frac{1}{2}$ & $\begin{array}{l}4 \\
1 \\
5 \\
1\end{array}$ & $\frac{3}{2}$ & $\frac{-}{-}$ & $\begin{array}{r}8(33,3 \%) \\
1 \quad(4,2 \%) \\
10(41,6 \%) \\
5(20,8 \%)\end{array}$ \\
\hline BAJO & $\begin{array}{l}\text { Matemát. } \\
\text { Sociales } \\
\text { Naturales } \\
\text { Lengua }\end{array}$ & $\begin{array}{l}\frac{1}{1} \\
-\end{array}$ & $\frac{\overline{2}}{\frac{1}{1}}$ & $\begin{array}{l}3 \\
\frac{5}{3}\end{array}$ & $\frac{-6}{2}$ & $\begin{array}{l}- \\
-\end{array}$ & $\begin{array}{r}3(12,5 \%) \\
15(62,5 \%) \\
-\quad- \\
6(25,0 \%)\end{array}$ \\
\hline
\end{tabular}

Valoración por materias de los profesores considerados mejor/peor inteligibles por parte de los alumnos sordos $(N=24)$.

La comparación establecida por materias y en base al nivel de comprensión oral alcanzado por cada alumno refleja una tendencia hacia la consideración que el profesorado que imparte las áreas de Naturales y Matemáticas suele mostrarse más fácilmente comprensible para el alumno; a diferencia de lo que sucede en las áreas de Lenguaje y, fundamentalmente, Sociales. No obstante, a medida que el nivel de comprensión del alumno mejora, las preferencias suelen ser más heterogéneas, lo que invita a pensar que, en este último caso, la elección se produce no tanto en función del contenido de la materia sino del estilo didáctico del profesor.

Una posible explicación de los resultados aquí vertidos podría ser el hecho de que el área de Naturales suele impartirse mediante la frecuente utilización del material visual que refleja diferentes aspectos de la vida más cercana del alumno: «El cuerpo humano»; «Los seres vivos»; «Las plantas»; «Los fenómenos geológicos»... Por el contrario, el área de conocimientos de Sociales, especialmente a partir de Segunda Etapa de EGB, se impregna de contenidos bastante abstractos para el alumno sordo y difíciles de comprender para aquellos con importantes carencias culturales: las nociones históricas, la política, la economía, el hábitat...

Por otra parte, en cierta medida resulta sorprendente la elección mayoritaria de los profesores de Matemáticas como colectivo de docentes más inteligible para el alumno, en tanto en cuanto esta área de conocimiento suele presentar importantes dificultades para el sordo. Estos resultados nos conducen a considerar el hecho que la tipología de las actividades académicas desarrolladas en Matemáticas respecto al sordo sea fundamentalmente la realización de ejercicios mecánicos, lo cual precisa de un tipo de consignas muy breves y estandarizadas que, si bien favorecen su comprensión, suponen un grado de dificultad cognitiva muy elemental. 
Los propios alumnos indican las principales razones por las cuales consideran a un determinado grupo de profesores como los menos inteligibles durante sus explicaciones. En primer lugar, destaca como la mayor parte de las opiniones $-51 \%$ - indican que la razón fundamental es la complejidad de la propia materia; a continuación, un $29 \%$ observa que el profesor habla excesivamente deprisa («habla oscura») y, por último, el $20 \%$ apela a la diferencia entre el habla del profesor (catalán o castellano) y la del propio alumno.

\section{Principales receptores de las demandas}

La Figura 4 refleja la distribución de las personas receptoras de las demandas de refuerzo por parte del alumno sordo, con posterioridad a la clase.

FIGURA 4

Area de conocimientos impartida por el profesor más inteligible

\begin{tabular}{|l|c|c|}
\hline \multirow{2}{*}{ RECEPTOR DEMANDA } & \multicolumn{2}{c|}{ OPINIONES } \\
\cline { 2 - 3 } & SORDO & PROFESOR \\
\hline Profesor del aula & $39 \%$ & $49 \%$ \\
Logopeda & $24 \%$ & $11 \%$ \\
Compañero de mesa & $34 \%$ & $31 \%$ \\
Familia & - & $9 \%$ \\
Ayuda externa & $3 \%$ & - \\
\hline$\Sigma$ & $100 \%$ & $100 \%$ \\
\hline
\end{tabular}

Area de conocimientos impartida por el profesdor menos inteligible

\begin{tabular}{|l|c|c|}
\hline \multirow{2}{*}{ RECEPTORES DEMANDAS } & \multicolumn{2}{|c|}{ OPINIONES } \\
\cline { 2 - 3 } & SORDO & PROFESOR \\
\hline Profesor del aula & $16 \%$ & $25 \%$ \\
Logopeda & $26 \%$ & $32 \%$ \\
Compañero de mesa & $39 \%$ & $44 \%$ \\
Familia & $13 \%$ & $8 \%$ \\
Ayuda externa & $6 \%$ & $1 \%$ \\
\hline$\Sigma$ & $100 \%$ & $100 \%$ \\
\hline
\end{tabular}

Principal receptor de las demandas del alumno sordo.

De la lectura de esta Figura podemos destacar el hecho de que, por regla general, los profesores considerados por el alumno como más comprensibles manifiestan mayor protagonismo al considerarse como principales receptores de las demandas del alumno durante las incomprensiones generadas en el aula; a diferencia de los profesores menos comprensibles, quienes otorgan mayor importancia al compañero de mesa del alumno. Al mismo tiempo, los profesores menos comprensibles otorgan bastante importancia al logopeda, delegándole gran parte de la responsabilidad como educador del alumno sordo. Estos datos son contrarios a los observados en relación a los profesores más comprensibles, quienes aparentemente conceden bastante menos protagonismo al logopeda.

La valoración efectuada por los propios alumnos sordos presenta unos resul- 
tados bastante semejantes, si exceptuamos la mejor valoración que el propio alumno hace tanto de las demandas dirigidas al logopeda como de las dirigidas a un refuerzo externo al centro escolar.

\section{Los elementos favorables de comprensión utilizados por el profesor}

En la Figura 5 aparece la distribución de la importancia concedida a los facilitadores contextuales utilizados por el oyente, en base a la opinión tanto del alumno como de los respectivos profesores. Los facilitadores contextuales considerados han sido:

1. Las adaptaciones morfosintácticas (enlentecer el ritmo, simplificar el léxico y la estructura del habla...).

2. Emplear el gesto natural para enfatizar determinados aspectos de la información a traspasar.

3. Utilizar material visual o manipulativo (escritura, dibujo, elaborar gráficos o esquemas sinópticos...).

4. Explicación adicional individualizada.

5. Anticipación de los contenidos básicos de la materia.

6. Solicitar los apuntes del compañero.

\section{Figura 5}

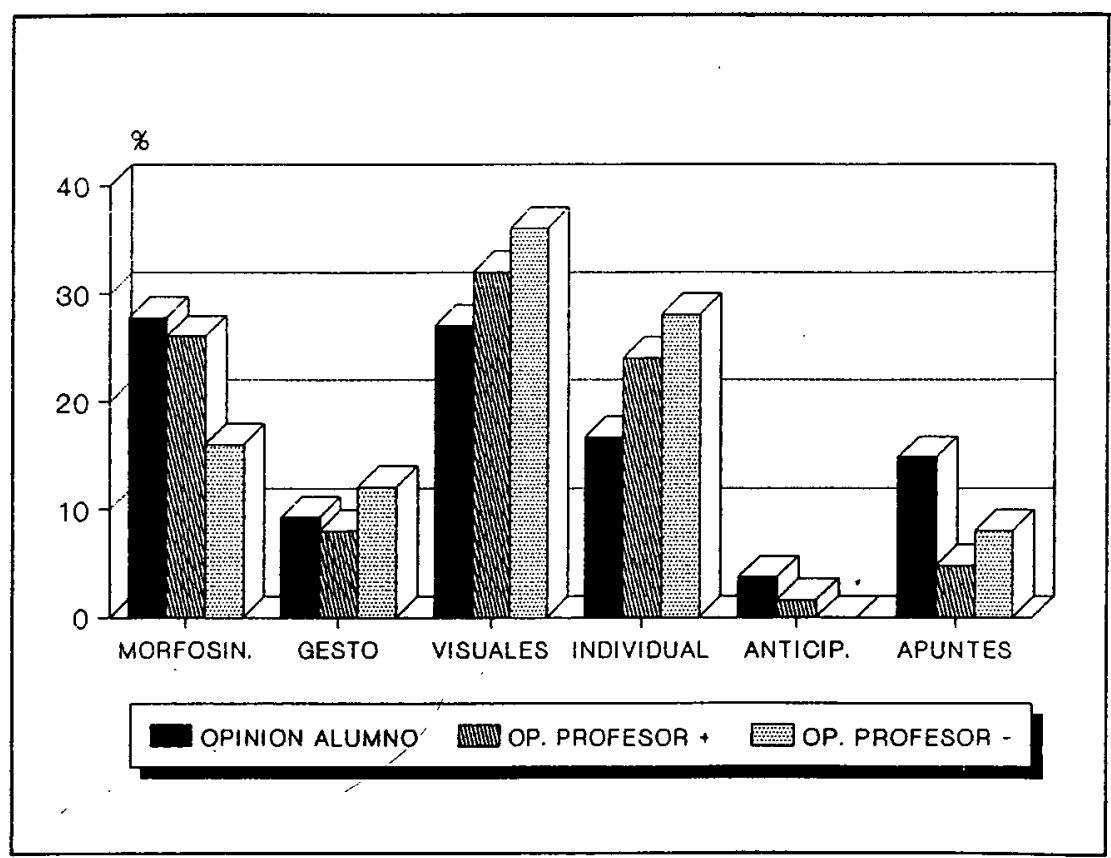

Elementos de ajuste favorables para la comprensión del alumno sordo.

Debemos valorar estos datos considerando la dependencia que existe entre el uso de determinados facilitadores y el área de conocimientos impartida por el profesor. En el sentido de que probablemente sea más sencillo utilizar facili- 
tadores contextuales de tipo visual o táctil en el área de Naturales o Sociales, a diferencia de las posibilidades que ofrece el Lenguaje, por ejemplo.

Complementando la anterior figura, la Figura 6 refleja cómo las preferencias por una u otra estrategia están estrechamente relacionadas con la capacidad de comprensión lingüística del alumno sordo.

FIGURA 6

\begin{tabular}{|l|c|c|}
\hline \multirow{2}{*}{ ELEMENTOS FAVORABLES } & \multicolumn{2}{|c|}{ NIVEL DE COMPRENSION } \\
\cline { 2 - 3 } & I y II & III, IV y V \\
\hline Adaptaciones morfosintácticas & $33,5 \%$ & $20,0 \%$ \\
Gesticulación/mímica & $19,0 \%$ & $5,8 \%$ \\
Material visual & $24,0 \%$ & $25,7 \%$ \\
Anticipación materia & $19,0 \%$ & $14,2 \%$ \\
Explicación individual & - & $11,4 \%$ \\
Apuntes del compañero & $4,5 \%$ & $22,9 \%$ \\
\hline$\Sigma$ & $100,0 \%$ & $100,0 \%$ \\
\hline
\end{tabular}

Comparación de las proporciones halladas entre los elementos favorables de comprensión del alumno sordo y el nivel de comprensión lingüistica del mismo.

Así, en los niveles más bajos, se vislumbra una tendencia hacia la preferencia de ayudas más dependientes del adulto, lo que en cierta medida recuerda las prácticas didácticas de las escuelas especiales de sordos: modificar el léxico, ayudarse del gesto, hablar más lentamente... No obstante, aquellos alumnos que mantienen un mejor nivel lingüístico tienden a establecer preferencias por un tipo de ayudas más normalizadas entre la población escolar oyente: utilizar los apuntes del compañero, solicitar una explicación individual posterior...

Por último cabe resaltar como la utilización de material visual —dibujos, mapas, escritura, esquemas sinópticos, subrayado...- suele ser aceptado mayoritariamente por todos los sujetos, independientemente de su nivel de comprensión lingüística.

Uno de los ámbitos sobre los que estamos trabajando en estos momentos en relación al uso de facilitadores contextuales por parte del oyente es el de su eficacia y su relación con las diversas estrategias empleadas para resolver los conflictos comunicativos en el aula: repetir la consigna, ayudar al alumno a encontrar la respuesta correcta, facilitar la respuesta directamente... - analizando, así mismo, las semejanzas respecto a la acción ejercida con el alumno oyente en situaciones afines (Silvestre, 1991; Valero, 1992).

Coincidiendo con la mayor parte de los estudios realizados durante la pasada década, los primeros datos apuntan hacia la consideración de que el tipo de estrategias empleadas por el profesor respecto al alumno sordo suelen ser más restrictivas que las empleadas frente al alumno oyente - repetir la consigna o dar la solución directamente-, si bien las causas que generan las incomprensiones al aula suelen también ser percibidas por el profesor de forma distinta. Así, para el alumno oyente destaca la necesidad de efectuar clarificaciones conceptuales y expansivas, mientras que en el sordo la necesidad de clarificaciones puntuales se concentra en la repetición de términos lingüísticos no conocidos o percibidos de forma irregular. Probablemente este hecho sea debido a que 
detrás de una demanda puntual de tipo léxico se suele ocultar una dificultad conceptual más general, pero difícil de percibir para el profesor poco habituado.

\section{DISCUSION}

Todo aprendizaje se realiza en un entorno social por medio de intercambios comunicativos en los que profesor y alumno construyen conjuntamente el conocimiento.

Las dificultades surgen cuando las reglas que regulan los códigos comunicativos son diferentes, obedeciendo a contextos de origen diverso. En estas situaciones suelen abundar las incomprensiones didácticas, lo que limita el acceso al conocimiento por parte del alumno, en nuestro caso, sordo.

Los primeros datos de nuestro estudio apuntan hacia la consideración de las siguientes implicaciones pedagógicas:

- La inclusión de estudiantes sordos en aulas regulares obliga al profesor a concebir, y en su caso modificar, un estilo docente fundamentado en los principios del aprendizaje individualizado y activo, donde la preparación del material, la presentación y el sistema de evaluación pueden ser intencional y sustancialmente modificados para facilitar el desarrollo académico y social del alumno sordo.

- La utilización del mayor número posible de indicadores visuales y el favorecer la acción tutorial del compañero oyente pueden resultar estrategias de trabajo muy válidas con el sordo. Del mismo modo, el facilitar resúmenes de cada temario, donde se destaquen los aspectos clave, el cerciorarse que el alumno ha comprendido las consignas y el facilitarle una ubicación en clase que le permita controlar visualmente el espacio y efectuar una correcta lectura labial son aspectos valorados muy positivamente por los estudiantes sordos.

- En otro orden de cosas, el estilo didáctico del profesor, la experiencia previa en educación diferenciada, el área de conocimientos impartida y la propia capacidad lingüística del alumno sordo parecen mantener, conjuntamente, una decisiva influencia sobre el grado de inteligibilidad de la acción docente, así como sobre la utilización de determinados facilitadores contextuales.

- Sin duda, la actual Reforma Educativa es un instrumento esencial para el tratamiento de las necesidades educativas especiales, al favorecer un planteamiento curricular abierto en el aula regular y la articulación de estrategias metodológicas más ajustadas a la particularidad comunicativa del deficiente auditivo.

- Proporcionar al profesor el conocimiento y la información necesarias para entender la especificidad del sordo, así como aquellos procedimientos que ayuden a mejorar su desarrollo pueden suponer un avance en el proceso de integración de estos alumnos. No obstante, son necesarias futuras investigaciones que ayuden a diseñar estrategias didácticas para una mejor formación del profesorado. 


\section{Referencias}

Aguilera, M. J., et al (1990). Evaluación del Programa de integración escolar de alumnos con defi. ciencia. Madrid: CIDE/Ministerio de Educación y Ciencia.

Dengra, M. R.; Durín, B., y Verdugo, A. (1991). Estudio de las variables que afectan a las actitudes de los maestros hacia la integración escolar de niños con necesidades especiales. Anuario Español e Iberoamericano de Investigación en Educación Especial. 2, 47-88.

Garcia, J. N., y Alonso, J. C. (1985). Actitudes de los maestros hacia la integración escolar de niños con necesidades especiales. Infancia y Aprendizaje. 30, 51-68.

Hartley, I., y Arnold, P. (1988). Communication of Hearing Impaired Student in Further Educationa. Journal. Britanish. Assn. Teachers of the Deaf. (12) 4, 73.76.

Hegarty, S.; Hodgson, A., y Clunies-Ross, L. (1986). Learning Together. Publicado en castellano: Aprender juntos. La integración escolar. Madrid: Morata, 1988.

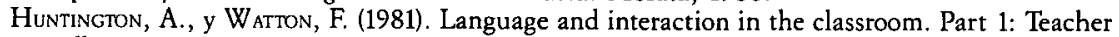
talk. Joumal Britanish Assn. Teachers of the Deaf. (5) 6, 163.173.

Huntington, A., y Whtron, F. (1982). Language and interaction in the classroom. Part 2: Pupil talk. Journal Britanish Assn. Teachers of the Deaf. (6) 1, 18-21.

Kretschimer, R., y Kretschmer, L. (1989). Communication Competence: Impacts of the Pragmatics Revolution on Education of Hearing Impaired individuals. Topics in Language Disorders. (9) $1,1-15$.

LYNAS, W. (1986). Integrating the handicapped into ordinary schools. Londres: Croom Helm.

MARChesI, A. (ed.) (1987). El desarrollo cognitivo y lingüístico de los niños sordos. Madrid: Alianza Psicología, 17.

Monereo, C., y Guardia, J. (1987). «Estrategias de intervención para la modificación de actitudes hacia la integración escolar». En Monereo, C. (eds.). Areas de intervención del Psicólogo de la Educación en la integración escolar del alumno con necesidades educativas excepcionales. Barcelona: Federación ECOM.

Parrillı, A. (1988). El pensament del professor sobre la integració. Guix. 125, 15-18.

Power, D.; WoOd, D., y WoOd, J. (1990). Conversation Strategies of Teacher using three Methods of Communication with Deaf Children. American Annals of the Deaf. 135, 9-13.

SAUR, R.E.; Popp, M., y HurLey, E. (1987). The Classroom participation of mainstreamed Hearingimpaired College students. The Volta Review. Oct/Nov. 277-286.

SILVESTRE, N. (1991). Las interacciones entre profesor y adolescente sordo profundo integrado en el aula regular con los oyentes. Revista de Logopedia, Foniatría y Audiologia. Vol XI, 3, 170-177.

VALERO, J. (1992). Les situacions d'incomprensió de l'adolescent sord durant els actes d'interacció comunicativa amb el professor de l'aula regular. Memoria de Investigación. Dpt. de Psicologia de l'Educació. Universidad Autónoma de Barcelona.

WEISS, A. L. (1986). Classroom discourse and the Hearing Impaired Child. Topics in Language Disorders. VI, 3, 60-70.

WOOD, D.; WoOD, H.; Griffrths, A., y HowArTh, I. (1986). Teaching and talking with deaf children. Chichester: Wiley. 


\section{Los elementos facilitadores de la comprensión oral empleados por el profesor del aula regular con adolescentes sordos. Jesús Valero CL\&E, 1993, 18, pp. 101-113}

Resumen: El alumno sordo precisa de un conjunto de estrategias específicas para superar satisfactoriamente las diversas situaciones comunicativas generadas en contextos académicos. Este compromiso obliga generalmente al profesor a emplear diversas adaptaciones - tanto pedagógicas como comunicativas - respecto a la actividad realizada en el aula. De entre estas posibles adaptaciones destacan las concebidas para solventar situaciones de incomprensión comunicativa.

$\mathrm{Si}$ bien el respeto por parte del oyente de aquellas peculiaridades en la comunicación con el sordo - articular con claridad, simplificar la estructura morfosintáctica... - no siempre resulta sencillo para el profesor poco familiarizado con el mundo del sordo, tampoco éste suele manifestar fácilmente sus dificultades de comprensión, lo que a menudo entorpece la labor docente del profesor.

Con el presente artículo se pretende analizar y establecer una posible relación entre las estrategias de acomodación utilizadas y un conjunto de variables tanto intrapersonales como contextuales, presentes durante las interacciones en el aula.

Datos del autor: Psicólogo. Logopeda. Unidad de Formación del CREDA-Comarcas 1. Departamento de Educación. Generalitat de Catalunya.

Trabajo en la integración en Centros Públicos de alumnos con déficit auditivo, tanto desde la intervención directa como desde la formación específica de profesores tutores. Mi área de interés profesional se centra en el estudio de la comunicación oral entre el alumno sordo y el profesor del aula regular.

Dirección: Jesús Valero. Unidad de Formación. CREDA. C/ Verdaguer, 3. 08500 VIC. Barcelona.

Agradecimientos: Este trabajo forma parte de un estudio más global dirigido por la doctora. Nuria Silvestre, profesora titular del Departamento de Psicología Básica de la Universidad Autónoma de Barcelona, abarcando diferentes aspectos del desarrollo psíquico y lingüístico del adolescente sordo. La investigación tiene el soporte económico de la DGICYT.

(C) De todos los artículos deberá solicitarse por escrito autorización de CL\&E y de los autores para el uso en forma de facsímil, fotocopia o cualquier otro medio de reproducción impresa. CL\&E se reserva el derecho de interponer las acciones legales necesarias en aquellos casos en que se contravenga la ley de derechos de autor. 